\title{
Equaçoes de referência para a predição da força de preensão manual em brasileiros de meia idade e idosos
}

\author{
Reference equations for predicting of handgrip strength in Brazilian middle-aged \\ and elderly subjects
}

\author{
Rômulo Dias Novaes, ${ }^{1}$ Aline Silva de Miranda², Jaqueline de Oliveira Silvaă, \\ Bruna Vasconcelos Fonseca Tavares ${ }^{3}$, Victor Zuniga Dourado ${ }^{4}$
}

Estudo desenvolvido na

Universidade Federal dos Vales

do Jequitinhonha e Mucuri,

Diamantina, MG, em

colaboração com a Unifesp -

Universidade Federal de São

Paulo, campus Baixada

Santista, Santos, SP, Brasil

1 Fisioterapeuta; mestrando em Biologia Celular e Estrutural no Depto. de Biologia Geral da Universidade Federal de Viçosa, Viçosa, MG

2 Fisioterapeuta; mestranda em Infectologia e Medicina

Tropical no Depto. de Ciências Básicas da Universidade Federal de Minas Gerais, Belo Horizonte, MG

3 Fisioterapeutas

4 Fisioterapeuta; Prof. Dr. adjunto do Depto. de Ciências da Saúde da Unifesp, Santos, SP

ENDEREÇO PARA

CORRESPONDÊNCIA

Victor Z. Dourado

Unifesp, Campus Baixada Santista

Av. Alm. Saldanha da Gama 89

11030-400 Santos SP

e-mail:

vzdourado@yahoo.com.br;

victor.dourado@unifesp.br

\section{APRESENTAÇÃo}

mar. 2009

ACEITO PARA PUBLICAÇÃO

jul. 2009
Resumo: O objetivo deste estudo foi avaliar os valores normais da força de preensão manual do membro superior dominante (FPM-D) e não dominante (FPM-ND) em sujeitos de meia idade e idosos assintomáticos e elaborar equações de referência para a predição da FPM. Foram investigados 54 voluntários (51,9\% homens) com idade $\geq 50$ anos, medindo-se massa corporal, estatura e perimetria do braço direito e esquerdo, e calculando-se o índice de massa corporal. A FPM-D e FPM -ND foram avaliadas por dinamometria mecânica. $\mathrm{O}$ índice de atividade física habitual (IAF) foi avaliado pelo questionário de Baecke. A FPM-D foi superior à FPM-ND em ambos os sexos e em todas as idades $(p<0,05)$. Foram encontradas correlações significativas entre a FPM e idade, estatura, massa corporal e perimetria do braço. As melhores equações de referência foram as seguintes:

FPM- $D_{\text {kgf }}=39,996-\left(0,382 \times\right.$ idade $\left._{\text {anos }}\right)+\left(0,174 \times\right.$ pesO $\left._{\mathrm{kg}}\right)+\left(13,628 \times\right.$ sexo $\left._{\text {homens }=1 ; \text { mulheres }=0}\right)$ ( $\mathrm{R}^{2}$ ajustado=0,677); e

FPM-ND kgf $=44,968-\left(0,420 \times\right.$ idade $\left._{\text {anos }}\right)+\left(0,110 \times\right.$ peso $\left._{\mathrm{kg}}\right)+\left(9,274 \times\right.$ sexo $\left._{\text {homens }=1 ; \text { mulheres }=0}\right)$ ( $\mathrm{R}^{2}$ ajustado=0,546)

A diferença consistente entre a FPM-D e FPM-ND torna necessário o uso de dados normativos específicos para cada mão. Atributos simples de serem obtidos, tais como idade, estatura, massa corporal, perimetria do braço e sexo, podem pois prever adequadamente os valores esperados da FPM para adultos e idosos assintomáticos.

Descritores: Antropometria; Força da mão; Medidas, métodos e teorias

ABSTRACT: The aim of the present study was to evaluate normal values of dominant (D) and non-dominant (ND) upper limb handgrip strength (HGS) in asymptomatic middle-aged elderly subjects and to establish reference equations for predicting HGS. Fifty-four volunteers ( $51.9 \%$ men) aged $\geq 50$ years old were enrolled. Weight, height, and both arms circumference were measured, being the body mass index calculated. Mechanical dynamometry was used to measure D-HGS and ND-HGS. Self-reported level of regular physical activity was assessed by Baecke questionnaire. D-HGS was higher than ND-HGS for both sexes and in all age groups $(p<0.05)$. Significant correlations were found between HGS and age, height, weight, and arm circumferences. The best reference equations were the following:

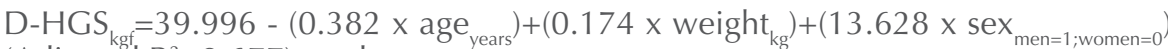
(Adjusted $\mathrm{R}^{2}=0.677$ ); and

ND-HGS kgf $=44.968-\left(0.420 \times\right.$ age $\left._{\text {years }}\right)+\left(0.110 \times\right.$ weight $\left._{\mathrm{kg}}\right)+\left(9.274 \times\right.$ sex $\left._{\text {men }=1 ; \text { women }=0}\right)$ (Adjusted $\mathrm{R}^{2}=0.546$ ).

The consistent difference found between dominant and non-dominant HGS requires the use of specific normative data for each hand. Hence easily-obtained attributes such as age, height, weight, arm circumference and sex can predict HGS expected values for asymptomatic elder adults.

KeY words: Anthropometry; Hand strength; Measurements, methods and theories 


\section{INTRODUÇÃO}

Tradicionalmente, testes de força de preensão manual (FPM) têm sido utilizados na reabilitação para avaliar a condição física dos membros superiores, por meio da mensuração da força dos músculos da mão e do antebraço ${ }^{1-5}$ de pacientes com diversas desordens na extremidade superior, decorrentes de artrite reumatóide, síndrome do túnel do carpo, epicondilite lateral, acidente vascular encefálico, lesões traumáticas e doenças neuromusculares ${ }^{1,2,6}$. A medida da FPM por dinamometria apresenta boa correlação com o nível funcional dos membros superiores e estado geral de saúde ${ }^{2,7,8}$, sendo amplamente utilizada na seleção de procedimentos terapêuticos e acompanhamento da reabilitação funcional2,6,7,9,10

Nos últimos anos, a dinamometria manual vem sendo utilizada em diversos campos da área da saúde ${ }^{5,8,11-16}$; mais recentemente, estudos clínicos e epidemiológicos têm-na empregado na avaliação funcional de indivíduos idosos e hospitalizados $8,11,17-19$. Além disso, a dinamometria manual fornece indicadores do impacto de diferentes programas de exercício sobre o aprimoramento da força dos músculos da mão e antebraço ${ }^{5,12}$. Em associação com outras variáveis, como consumo de oxigênio e freqüência cardíaca máximos, a FPM pode ser utilizada como indicador de desempenho físico em modalidades esportivas que utilizam predominantemente os membros superiores, como escalada e tênis ${ }^{13-16}$.

A dinamometria manual apresenta ampla aplicabilidade, pois é um método de baixo custo, simples, rápido e nãoinvasivo $^{3,9,18,19}$, que fornece, por meio dos valores de FPM, um indicador da saúde geral dos indivíduos avaliados ${ }^{8,11,17}$. Estudos prévios mostraram que a dinamometria é capaz de auxiliar a detecção precoce de alterações metabólicas musculares como a redução da atividade do complexo mitocondrial, que leva à diminuição da produção de energia pelas células e, conseqüentemente, da capacidade dos músculos de gerar força ${ }^{9,20,21}$. Nesse contexto, os testes de FPM podem fornecer, em curto prazo, uma avaliação funcional adequada do estado e da recuperação energética do indivíduo, na presença ou até mesmo ausência de doença ${ }^{8,9,11,20}$.
A principal limitação da avaliação da FPM por meio da dinamometria é a ausência de um ponto de corte que defina o limite entre normalidade e disfunção para populações específicas ${ }^{8,11}$. A análise dos valores de referência apresentados em estudos prévios indica grande variação dos dados de FPM ${ }^{17-19}$. Há evidências de que essa variação ocorre devido às diferenças raciais, demográficas, antropométricas, sociais e culturais entre as amostras populacionais avaliadas nos diferentes estudos ${ }^{8,10,18}$. Dessa forma, para fornecer uma avaliação e acompanhamento mais adequados da função muscular, torna-se importante desenvolver instrumentos capazes de predizer os valores de FPM esperados para populações específicas.

O presente estudo levanta a hipótese de que os padrões de normalidade para a FPM da população adulta e idosa brasileira são provavelmente distintos dos apresentados por população estrangeira. Tendo em vista a grande aplicabilidade da FPM na avaliação física e funcional e a ausência de valores de referência para a população brasileira com idade igual ou superior a 50 anos, o presente estudo foi delineado com os seguintes objetivos: (1) avaliar os valores normais da FPM bilateralmente em indivíduos brasileiros com idade $\geq 50$ anos; e (2) elaborar, por meio de atributos demográficos e antropométricos, equações de referência para a previsão da FPM normal nesses indivíduos.

\section{METODOLOGIA}

Este é um estudo transversal, que avaliou 54 indivíduos saudáveis brasileiros com idade igual ou maior que 50 anos, de ambos os sexos. Os critérios de exclusão foram: doença sistêmica ou quadro álgico nos membros superiores, restrição em alguma atividade diária decorrente de doença, uso de substâncias estimulantes que interferissem na FPM, indivíduos ambidestros e não-compreensão do procedimento. Todos os voluntários foram esclarecidos oralmente e por escrito quanto aos objetivos e possíveis desconfortos relacionados ao presente estudo. Aqueles que concordaram em participar assinaram um termo de consentimento livre e esclarecido. O pre- sente estudo obteve aprovação do Conselho de Ética da Universidade Federal de São Paulo.

Peso corporal e estatura foram mensurados e o índice de massa corporal (IMC) foi calculado [IMC = peso corporal $(\mathrm{kg}) /$ altura $\left.\left(\mathrm{m}^{2}\right)\right]^{22}$. O índice de atividade física habitual (IAF) dos voluntários foi estimado por meio do questionário de Baecke, validado para a população brasileira $^{23}$. A FPM foi mensurada por meio de dinamômetro mecânico (Kratos, Cotia, SP, Brasil). A calibração do dinamômetro foi verificada periodicamente durante o estudo. Foi medido o perímetro de cada braço no ponto médio entre o acrômio da escápula e o epicôndilo lateral do úmero. Três medidas foram obtidas e a média aritmética dos valores foi utilizada para a análise dos dados.

A avaliação da FPM foi feita por um único investigador previamente treinado, no período da manhã entre as 8 e 12 horas, de acordo com as recomendações da Sociedade Brasileira de Terapeutas da Mão ${ }^{24}$. Os indivíduos foram posicionados sentados com o braço aduzido paralelo ao tronco, ombro em rotação neutra, cotovelo flexionado a $90^{\circ}$, antebraço em posição fundamental. Hiperextensão do punho de até $30^{\circ}$ e desvio ulnar de até $15^{\circ}$ foram permitidos durante os testes ${ }^{3,18}$. Foram tomadas três medidas de ambas as mãos, com intervalo mínimo de 30 segundos entre elas. Os testes foram realizados alternadamente entre o lado dominante e não-dominante, para minimizar a influência da fadiga muscular. O maior valor obtido para cada mão foi submetido à análise dos dados ${ }^{10,19}$. A mão dominante foi definida como a preferida para a realização das atividades diárias. Os voluntários foram inquiridos sobre a mão de preferência para a realização de atividades como escrever, comer e carregar objetos. Durante a execução da preensão manual, o braço permaneceu imóvel, havendo somente permissão para a movimentação das articulações do punho e dedos.

Por meio do pacote estatístico SigmaStat 2.03 (SPSS, Chicago, IL, USA) foram feitas as seguintes análises estatísticas: Kolmogorov-Smirnov para análise da normalidade na distribuição das variáveis investigadas; medidas de ten- 
dência central (média \pm desvio-padrão) para análise descritiva dos dados; e coeficientes de Pearson ou Spearman para avaliar as correlações entre as variáveis. Adicionalmente, foi utilizado o teste $t$ de Student para comparar os valores médios das variáveis entre homens e mulheres e entre FPM da mão dominante (FPM-D) e da mão nãodominante (FPM-ND) na amostra total, para comparar os valores médios da FPM entre homens e mulheres estratificados por faixa etária e para comparar esses valores entre o lado dominante e nãodominante em cada faixa etária. A multicolinearidade foi evitada removendo-se do modelo estatístico variáveis com alta correlação entre si ( $r>0,70$ ou $r<-0,70)$ e as variáveis com fator de inflação de variância VIF $>4$. Para as variáveis com associação maior que a supracitada, foi selecionada aquela de maior relevância clínica.

\section{RESULTADOS}

As características gerais da amostra estudada estão apresentadas na Tabela 1. Participaram do estudo 54 indivíduos, $28(51,9 \%)$ homens e $26(48,1 \%)$ muIheres. Todos os voluntários apresentaram dominância direita. Os homens apresentaram valores de peso e estatura significativamente superiores aos das mulheres (Tabela 1).

Em média os indivíduos apresentaram IMC dentro dos padrões de normalidade. Uma voluntária $(1,8 \%)$ apresentou baixo peso, 12 homens e 10 mulheres $(40,7 \%)$ eram eutróficos, 15 homens e 15 mulhe-
Tabela 2 Força de preensão manual (kgf) nas mãos dominante e não-dominante (média, desvio padrão, valores mínimos e máximos e erro padrão), nos homens $(n=28)$ e mulheres $(n=26)$ da amostra, segundo a faixa etária

\begin{tabular}{|c|c|c|c|c|c|c|c|c|c|c|c|}
\hline \multirow{2}{*}{ Idade } & \multirow{2}{*}{ Mão } & \multicolumn{5}{|c|}{ Homens } & \multicolumn{5}{|c|}{ Mulheres } \\
\hline & & Média & $d p$ & $\mathrm{EP}$ & Máx & Mín & Média & $d p$ & $\mathrm{EP}$ & Máx & Mín \\
\hline \multirow{2}{*}{ 50-59 } & $\mathrm{D}$ & 47,1 & 5,1 & 1,7 & 58 & 41 & 31,3 & 5,0 & 1,5 & 39 & 24 \\
\hline & ND & 42,8 & 5,1 & 1,7 & 50 & 36 & 29,3 & 5,3 & 1,7 & 38 & 19 \\
\hline \multirow{2}{*}{$60-69$} & $\mathrm{D}$ & 41,1 & 9,4 & 2,8 & 67 & 30 & 23,8 & 3,0 & 1,0 & 28 & 20 \\
\hline & ND & 33,7 & 8,3 & 2,5 & 50 & 18 & 23,0 & 2,5 & 0,8 & 26 & 18 \\
\hline \multirow{2}{*}{$70+$} & $\mathrm{D}$ & 37,4 & 5,6 & 1,8 & 46 & 30 & 23,8 & 7,2 & 2,9 & 31 & 14 \\
\hline & ND & 30,8 & 5,5 & 1,8 & 41 & 23 & 22,6 & 5,0 & 2,0 & 29 & 14 \\
\hline \multirow{2}{*}{ otal } & $\mathrm{D}$ & 41,9 & 8,4 & 1,0 & 67 & 30 & 26,3 & 6,9 & 0,9 & 39 & 14 \\
\hline & ND & 35,8 & 9,5 & 1,2 & 50 & 18 & 25,0 & 6,7 & 0,8 & 38 & 14 \\
\hline
\end{tabular}

DP = desvio-padrão; EP = erro-padrão; Máx = valor máximo; Mín = valor mínimo; D = dominante; ND = não dominante

res $(55,5 \%)$ apresentaram sobrepeso e uma voluntária $(1,8 \%)$ apresentou obesidade grau I. Quando analisado o índice de atividade física (IAF), oito homens e seis mulheres (26\%) alcançaram resultado superior a 8 , sendo o restante dos indivíduos, 20 homens e 20 mulheres $(74 \%)$, considerados sedentários $(\mathrm{IAF} \leq 8)$.

A estatística descritiva da FPM na amostra estudada é apresentada na Tabela 2. Os valores médios da FPM foram maiores para a mão dominante em relação à mão não- dominante $(35 \pm 10$ kgf vs $30 \pm 8 \mathrm{kgf} ; p<0,05)$. Os homens apresentaram valores médios da FPM dominante superiores aos das mulheres (respectivamente $41 \pm 8$ kgf vs. $26 \pm 7$ kgf; $p<0,05)$.

A FPM do membro superior dominante (no caso, o direito, em toda a amostra) apresentou correlações significativas, moderadas a fortes, com a idade, esta-

Tabela 1 Características gerais da amostra $(n=54)$

\begin{tabular}{|c|c|c|c|c|c|c|c|c|}
\hline \multirow{2}{*}{ Característica } & \multicolumn{4}{|c|}{ Homens } & \multicolumn{4}{|c|}{ Mulheres } \\
\hline & $50-59$ & $60-69$ & $70+$ & Total & $50-59$ & $60-69$ & $70+$ & Total \\
\hline $\mathrm{N}$ & 9 & 10 & 9 & 28 & 10 & 9 & 7 & 26 \\
\hline Idade (média, anos) & $54 \pm 4$ & $64 \pm 3$ & $73 \pm 4$ & $64 \pm 9$ & $53 \pm 3$ & $65 \pm 2$ & $74 \pm 3$ & $64 \pm 11$ \\
\hline Estatura (cm) & $173 \pm 6$ & $171 \pm 5$ & $169 \pm 6$ & $171 \pm 2$ & $159 \pm 4$ & $152 \pm 6$ & $151 \pm 8$ & $154 \pm 4^{*}$ \\
\hline Peso (kg) & $83 \pm 8$ & $69 \pm 9$ & $70 \pm 13$ & $74 \pm 8$ & $63 \pm 9$ & $65 \pm 10$ & $59 \pm 8$ & $62 \pm 3^{*}$ \\
\hline IMC $\left(\mathrm{kg} / \mathrm{m}^{2}\right)$ & $27 \pm 1$ & $23 \pm 3$ & $24 \pm 3$ & $25 \pm 2$ & $24 \pm 2$ & $28 \pm 5$ & $25 \pm 2$ & $26 \pm 2$ \\
\hline PMSD (cm) & $32 \pm 2$ & $30 \pm 2$ & $29 \pm 3$ & $30 \pm 1$ & $29 \pm 2$ & $30 \pm 4$ & $29 \pm 2$ & $29 \pm 1$ \\
\hline PMSND (cm) & $32 \pm 1$ & $29 \pm 2$ & $29 \pm 3$ & $30 \pm 2$ & $28 \pm 2$ & $29 \pm 4$ & $29 \pm 2$ & $29 \pm 1$ \\
\hline IAF & $8 \pm 2$ & $6 \pm 2$ & $6 \pm 2$ & $7 \pm 1$ & $8 \pm 2$ & $6 \pm 2$ & $6 \pm 1$ & $7 \pm 1$ \\
\hline
\end{tabular}

IMC = índice de massa corporal; PMSD = perímetro do membro superior dominante; PMSND = perímetro do membro superior não-dominante; IAF = índice de atividade física habitual de Baecke; ${ }^{*}=p<0,05$ homens vs mulheres tura, peso e perimetria do braço direito (Figura 1).

A FPM-ND apresentou correlações significativas, moderadas a intensas, com a idade $(r=0,42 ; p<0,01)$, estatura $(r=0,55 ; p<0,001)$,, peso $(r=0,49$; $p<0,001)$ e perimetria do braço esquerdo, não-dominante em toda a amostra $(r=0,33 ; p=0,0144)$. Observou-se correlação significativa entre a FPM-ND e o escore do domínio relacionado às atividades de lazer do IAF $(r=0,27$; $p=0,04$ ).

As equações de referência para a predição da FPM-D e FPM-ND são apresentadas abaixo. Note-se que idade, peso corporal e sexo foram responsáveis por $67,7 \%$ da variância total da FPM-D e as mesmas variáveis foram responsáveis por $54,6 \%$ da variância total da FPM-ND. A adição da estatura e da perimetria do braço não aumentou significativamente o poder de predição das equações de regressão.

Equações para a previsão da FPM-D (em kgf):

- $F P M-D=39,996-(0,382 \times$ Idade $)$ $+(0,174 \times$ Peso $)+(13,628 \times$ Sexo $*)$; $\mathrm{R}^{2}$ Ajustado $=0,677 ; \mathrm{EPE}=5,917$ kgf

- $\mathrm{FPM-D}=29,099-(0,363 \times$ Idade $)$ $+(0,0681 \times$ Estatura $)+(0,160 \times$ Peso $)+\left(12,658 \times\right.$ Sexo* $\left.^{*}\right) ; R^{2}$ Ajustado $=0,672 ; \mathrm{EPE}=5,965 \mathrm{kgf}$

- $\mathrm{FPM}-\mathrm{D}=20,591-(0,368 \times$ Idade $)+$ $(0,0941 \times$ Estatura $)+(0,0723 \times$ Peso $)$ $+(0,350 \times$ perimetria do braço dominante $)+\left(12,748 \times\right.$ Sexo $\left.^{*}\right) ; R^{2}$ 

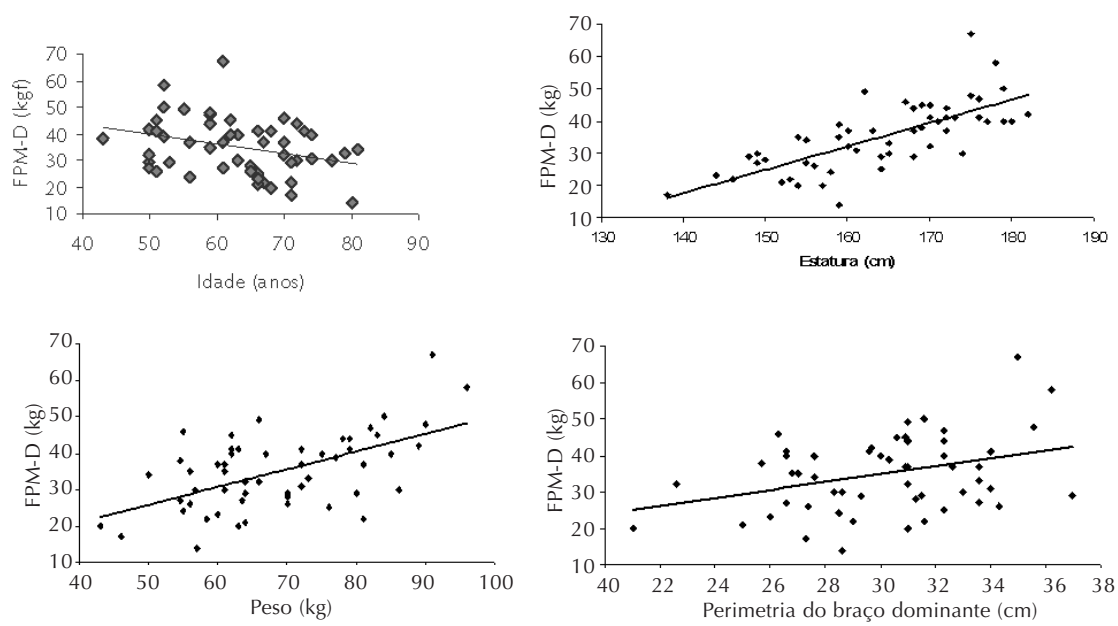

Figura 1 Correlações significativas entre força de preensão manual do membro superior dominante (FPM-D) e idade $(r=0,31 ; p=0,0218)$, estatura $(r=$ $0,72 ; p<0,0001)$, peso $(r=0,57 ; p<0,0001)$ e perimetria do braço dominante $(r=0,35 ; p<0,01)$

Ajustado $=0,672 ; \mathrm{EPE}=5,967 \mathrm{kgf}$

Equações para a previsão da FPM-ND (em kgf):

- $F P M-N D=44,968-(0,420 \times$ Idade $)$ $+(0,110 \times$ Peso $)+(9,274 \times \text { Sexo })^{*}$ $\mathrm{R}^{2}$ Ajustado $=0,546 ; \mathrm{EPE}=5,807$ kgf

- $F P M-N D=74,086-(0,472 \times$ Idade $)$ - $(0,182 \times$ Estatura $)+(0,148 \times$ Peso $)$ $+(11,866 \times$ Sexo $*) ; R^{2}$ Ajustado $=$ 0,$551 ; \mathrm{EPE}=5,776 \mathrm{kgf}$

- $F P M-N D=61,172-(0,479 \times$ Idade $)$ - $(0,146$ x Estatura $)+(0,0265 \times$ Peso $)+(0,526 \times$ perimetria do braço não dominante $)+(12,167 \times$ Sexo*); $R^{2}$ Ajustado $=0,559 ; \mathrm{EPE}=$ $5,728 \mathrm{kgf}$

A FPM, em quilograma força, deve ser calculada utilizando-se as seguintes unidades: idade em anos, peso em quilogramas, estatura em centímetros, perimetria do braço em centímetros, fator sexo* igual a 1 para os homens e 0 para as mulheres; e erro-padrão da estimativa (EPE) em quilograma força.

\section{DISCUSSÃO}

Os resultados encontrados mostram que a FPM-D e FPM-ND podem ser adequadamente preditas por meio de atributos demográficos e antropométri$\cos (67,7 \%$ e $54,6 \%$ da variância total, respectivamente). Observou-se, em consonância com a literatura prévia, que a FPM da mão dominante apresenta valores médios significativamente superiores aos encontrados para a mão não-dominante ${ }^{7,8,11,25}$. Adicionalmente, observou-se alta prevalência de sedentarismo na amostra estudada; contudo, houve influência discreta desse atributo na FPM.

A amostra estudada apresentou características demográficas e antropométricas similares às descritas para a população brasileira em geral. Foi encontrada prevalência de $57,3 \%$ de sobrepeso ou obesidade e de $74,0 \%$ para sedentarismo. Estudos epidemiológicos demonstraram que aproximadamente 1/3 da população brasileira na faixa etária $>50$ anos se encontra acima do peso ${ }^{26,27}$. Resultados semelhantes são descritos para o sedentarismo, que apresenta entre $70,9 \%$ e $79,5 \%$ de prevalência na faixa etária idosa ${ }^{28,29}$.

Os resultados do presente estudo mostram que os valores de FPM foram maiores no lado dominante para ambos os sexos em todas as faixas etárias estudadas. Esse achado pode ser explicado, em parte, pela maior freqüência de utilização do membro dominante em atividades diárias que exigem força, enquanto o membro contralateral é requerido principalmente em tarefas que envolvem coordenação fina. Esse padrão de utilização dos membros superiores permite desenvolver estratégias motoras e adaptações morfofisiológicas que implicam maior FPM no lado dominante, como hipertrofia muscular e maior recrutamento de unidades motoras $^{30,31}$. Valores de FPM cerca de 10\% maiores para a mão dominante têm sido evidenciados em ambos os $\operatorname{sexos}^{7,10,25}$. Tradicionalmente, tem sido utilizado o índice de $10 \%$ para estimar a FPM da mão não-dominante com base nos valores da mão dominante $e^{3,7,18}$. No presente estudo, a FPM-ND foi em média 7\% inferior à FPM-D, valores próximos dos descritos na literatura nessa faixa etária ${ }^{3,7,18}$. Entretanto, evidências atuais não permitem aplicar essa regra ao determinar alteração clínica da FPM, uma vez que a força de preensão pode ser influenciada por fatores como atividades laborais e hábitos sociais ${ }^{8,10}$.

A FPM sofreu influência significativa de variáveis demográficas e antropométricas. A FPM dos homens, como esperado, foi significativamente maior que a encontrada para as mulheres em todas as faixas etárias estudadas tanto para a mão dominante quanto para a não-dominante. Variações na FPM entre os gêneros têm sido atribuídas às diferenças na composição corporal entre homens e mulheres. Em indivíduos eutróficos, a massa muscular dos homens e das mulheres corresponde a aproximadamente $45 \%$ e $30 \%$ da massa corporal total, respectivamente. Além disso, embora o número de fibras musculares seja semelhante entre os sexos, a FPM sofre a influência do tamanho das fibras musculares, que é cerca de 15\% menor nas mulheres ${ }^{17,32}$.

A FPM apresentou correlações com o peso corporal e a estatura, indicando que essas variáveis são importantes para sua predição ${ }^{8,11,17}$. Innes ${ }^{10}$ reforça esses achados e afirma que a correlação positiva entre essas variáveis permanece mesmo nos extremos de estatura (190 cm) e peso corporal (98 kg). O peso corporal e a estatura, além da perimetria do braço, estão relacionados com a distribuição de massa muscular no braço e mão, influenciando, dessa forma, o tamanho da mão e, conseqüentemente, a alavanca relacionada à FPM. O maior peso corporal e estatura dos homens contribuem para uma grande área de secção transversa da musculatura do 
braço e mão, o que permite maior geração de força ${ }^{10,17}$. Contudo, os detalhes dessa relação requerem maiores esclarecimentos, tendo em vista a influência de outros fatores como o nível de atividade física habitual e a capacidade das células de produzir energia para a contração muscular ${ }^{1,20,21}$.

Foi encontrada correlação negativa fraca entre idade e FPM. Esse achado indica que outros atributos devem ser considerados na predição da FPM, uma vez que a idade, isoladamente, não foi capaz de explicar o declínio na FPM ao longo do tempo. Há dados suficientes na literatura segundo os quais a correlação entre FPM e idade é curvilinear, com o pico de força entre 20 e 50 $\operatorname{anos}^{8,11,17,18}$, o que torna as equações de regressão problemáticas para a previsão da FPM. Todavia, a partir dos 50 anos a FPM sofre declínio linear ${ }^{13,27}$. Além disso, a FPM é diretamente proporcional à idade até os 32 anos, tornando-se inversamente proporcional após essa ida$\mathrm{de}^{6,8,11,17}$. Nesse contexto, a substituição parcial das fibras musculares por tecido fibrogorduroso e a redução da ativação das unidades motoras são descritas como importantes fatores que contribuem para diminuição da FPM, principalmente em indivíduos sedentários ${ }^{4,17,30,31}$. O presente estudo avaliou indivíduos com idade igual ou superior a 50 anos para elaboração das equações de referência. Isso elimina a tendência curvilinear da relação entre FPM e idade encontrada para amplas faixas etárias e reduz possível viés na aplicabilidade das equações aqui apresentadas.
Observou-se correlação positiva fraca entre a FPM-ND e o escore do domínio relacionado às atividades de lazer do IAF. Não foram encontradas pesquisas estudando a relação entre o nível de atividade física habitual e a FPM em indivíduos não-atletas. O IAF foi considerado uma possível variável de influência nos valores de FPM, uma vez que reflete a condição física e a capacidade de realizar atividades diárias do indivíduo ${ }^{23}$. Adultos sedentários apresentam uma diminuição de $20 \%$ a $40 \%$ da massa muscular em relação a indivíduos treinados, o que indica uma grande perda na força muscular ${ }^{4}$. Há evidências de que a prática regular de exercícios físicos melhora o metabolismo energético celular e previne reduções acentuadas na força muscular e na capacidade de realizar atividades diárias ${ }^{4,5}$. Em consonância, estudo prévio demonstrou que mesmo a prática de exercícios físicos não-específicos para o membro superior previne perdas na $\mathrm{FPM}^{5}$.

O presente estudo apresenta limitações. A primeira diz respeito ao número reduzido de indivíduos avaliados. Entretanto, as equações envolveram o máximo de cinco variáveis independentes. O número de indivíduos avaliados, aproximadamente 10 vezes maior que o número de variáveis independentes, associado aos valores de $\mathrm{R}^{2}$ expressivos tornam as equações apresentadas úteis para a previsão da FPM. Adicionalmente, o quanto a amostra do presente estudo é representativa da população brasileira merece maiores esclarecimentos. Limitação semelhante foi descrita por
Mathiowetz et al. ${ }^{18}$ em estudo envolvendo indivíduos americanos. Finalmente, o uso do dinamômetro mecânico em vez do dinamômetro hidráulico, reconhecidamente mais acurado para a medida da FPM, pode ter influenciado os resultados encontrados. Contudo, desconhecemos estudos na literatura comparando esses dois dinamômetros especificamente. Em muitos centros de reabilitação brasileiros dispõese apenas de dinamômetro mecânico, o que torna os valores e equações de referência obtidas por meio desse instrumento relevantes.

\section{CONCLUSÃO}

Pode-se concluir que atributos simples de serem obtidos e amplamente empregados na avaliação física, tais como sexo, idade, estatura, peso corporal e perimetria do braço podem prever adequadamente os valores esperados da FPM do membro superior dominante e nãodominante para adultos brasileiros assintomáticos com idade igual ou superior a 50 anos. Tendo em vista a diferença consistente de força entre os lados dominante e não-dominante, torna-se prudente o uso de dados normativos específicos para ambos os membros superiores. Embora ainda não esteja bem estabelecido o ponto de corte entre a normalidade e a disfunção da FPM, acredita-se que as equações desenvolvidas no presente estudo podem auxiliar na avaliação do impacto de programas específicos de treinamento na FPM de indivíduos com ou sem doença e fornecer indicadores funcionais de alterações do metabolismo energético muscular.

\section{REFERÊNCIAS}

1 Kurillo G, Zupan A, Bajd T. Force tracking system for the assessment of grip force control in patients with neuromuscular diseases. Clin Biomech (Bristol, Avon). 2004;19(10):1014-21.

2 Figueiredo IM, Sampaio RF, Mancini MC, Nascimento MC. Ganhos funcionais e sua relação com os componentes de função em trabalhadores com lesão de mão. Rev Bras Fisioter. 2006;10(4):421-7.
3 Mathiowetz V, Rennells C, Donahoe L. Effect of elbow position on grip and key pinch strength. J Hand Surg. 1985;10(5):694-7.

4 Singh MAF. Exercise and aging. Clin Geriatr Med. 2004;20(2):201-21.

5 Rebelatto JR, Calvo JI, Orejuela JR, Portillo JC. Influência de um programa de atividade física de longa duração sobre a força muscular manual e a flexibilidade corporal de mulheres idosas. Rev Bras Fisioter. 2006;10(1):127-32. 


\section{Referências (cont.)}

6 Moreira D, Alvarez RRA, Gogoy JR, Cambraia AN. Abordagem sobre preensão palmar utilizando o dinamômetro Jamar: uma revisão de literatura. Rev Bras Cienc Mov. 2003;11(2):95-9.

7 Caporrino FA, Faloppa F, Santos JBG, Réssio C, Soares FHC, Nakachima LR, et al. Estudo populacional da força de preensão palmar com dinamômetro Jamar. Rev Bras Ortop Traumatol. 1998;33(2):150-5.

8 Schlüssel MM, Anjos LA, Kac G. A dinamometria manual e seu uso na avaliação nutricional. Rev Nutr. 2008;21(2):223-35.

9 Norman K, Schütz T, Kemps M, Lübke HJ, Lochs H, Pirlich M. The subjective global assessment reliably identifies malnutrition-related muscle disfunction. Clin Nutr. 2005;24(1):143-50.

10 Innes E. Handgrip strength testing: a review of the literature. Austr Occup Ther J. 1999;46(3):20-40.

11 Schlüssel MM, Anjos LA, Vasconcellos TL, Kac G. Reference values of handgrip dynamometry of healthy adults: a population-based study. Clinical Nutr. 2008;27(4):601-7.

12 Hansen L, Bangsbo J, Twisk J, Klausen K. Development of muscle strength in relation to training level and testosterone in young male soccer players. J Appl Physiol. 1999;87(3):1141-7.

13 Mermier CM, Janot JM, Parker DL, Swan JG. Physiological and anthropometric determinants of sport climbing performance. Br J Sports Med. 2000;34(5):359-65.

14 Cohen DB, Mont MA, Campbell KR, Barry VN, Loewy JW. Upper extremity physical factors affecting tennis serve velocity. Am J Sports Med. 1994; 22(6):746-50.

15 Kovacs MS. Applied physiology of tennis performance. Br J Sports Med. 2006;40(5):381-6.

16 Wall CB, Starek JE, Fleck SJ, Byrnes WC. Prediction of indoor climbing performance in women rock climbers. J Strength Cond Res. 2004;18(1):77-83.

17 Budziareck MB, Duarte RRP, Barbosa-Silva MCG. Reference values and determinants for handgrip strength in healthy subjects. Clin Nutr. 2008;27(3):357-62.

18 Mathiowetz V, Kashman N, Volland G, Weber K, Dowe M, Rogers S. Grip and pinch strength: normative data for adults. Arch Phys Med Rehabil. 1985;66:69-72.

19 Günther CM, Bürger A, Rickert M, Crispin A, Schulz $\mathrm{CU}$. Grip strength in healthy caucasian adults: reference values. J Hand Surg. 2008;33(4):558-65.
20 Figueiredo FA, Dickson ER, Pasha TM, Porayko MK, Therneau TM, Malinchoc M, et al. Utility of standard nutritional parameters in detecting body cell mass depletion in patients with end-stage liver disease. Liver Transpl. 2000;6(5):575-81.

21 Madapallimattam AG, Law L, Jeejeebhoy KN. Effect of hypoenergetic feeding on muscle oxidative phosphorilation and mitochondrial complex I-IV activities in rats. Am J Clin Nutr. 2002;76(5):1031-9.

22 Coutinho WF. Consenso latino-americano de obesidade. Arq Bras Endocrinol Metab. 1999;43(1):21-67.

23 Florindo AA, Latorre MRDO. Validação e reprodutibilidade do questionário de Baecke de avaliação da atividade física habitual em homens adultos. Rev Bras Med Esporte. 2003;9(3):121-8.

24 SBTM - Sociedade Brasileira de Terapeutas da Mão. Recomendações para avaliação do membro superior. São Paulo; 2008 [citado jul 2008]. Disponível em: www.sbtm.org.br.

25 Mathiowetz V, Weber K, Volland G, Rashman N. Reliability and validity of grip and pinch strength evaluations. J Hand Surg. 1984;9(2):222-6.

26 Cabrera MAS, Filho WJ. Obesidade em idosos: prevalência, distribuição e associação com hábitos e comorbidades. Arq Bras Endocrinol Metab. 2001;45(5):494-501.

27 Tavares EL, Anjos LA. Perfil antropométrico da população idosa brasileira: resultados da pesquisa nacional sobre saúde e nutrição. Cad Saude Publica. 1999;15(4):759-68.

28 Zaitune MPA, Barros BAB, César CLG, Carandina L, Goldbaum M. Fatores associados ao sedentarismo no lazer em idosos, Campinas, São Paulo, Brasil. Cad Saude Publica. 2007;23(6):1329-38.

29 Pitanga FJG, Lessa I. Prevalência e fatores associados ao sedentarismo no lazer em adultos. Cad Saude Publica. 2005;21(3):870-7.

30 Jensen JL, Marstrand PC, Nielsen JB. Motor skill training and strength training are associated with different plastic changes in the central nervous system. J Appl Physiol. 2005;99(4):1558-68.

31 Incel NA, Ceceli E, Durukan PB, Erdem HR, Yorgancioglu ZR. Grip strength: effect of hand dominance. Singapore Med J. 2002;43(5):234-7.

32 Stoll T, Huber E, Seifert B, Michel BA, Stucki G. Maximal isometric muscle strength: normative values and genderspecific relation to age. Clin Rheumatol. 2000;19(2):105-13. 